\title{
Observing inter-professional videos: impact of collaboration between physicians and psychologists on attitude and knowledge acquisition
}

\section{Johannes Großer}

Leibniz-Institut fur Wissensmedien / Knowledge Media Research Center

Joachim Kimmerle ( $\sim$ j.kimmerle@iwm-tuebingen.de )

Leibniz-Institut fur Wissensmedien / Knowledge Media Research Center https://orcid.org/0000-00026345-9498

\section{Thomas Shiozawa}

Universität Tübingen

\section{Bernhard Hirt}

Univerität Tübingen

\section{Martina Bientzle}

Leibniz-Institut fur Wissensmedien / Knowledge Media Research Center

\section{Research article}

Keywords: Inter-professional collaboration, inter-professional education, medical students, video, experimental study, deep brain stimulation, Parkinson's disease

Posted Date: April 27th, 2020

DOI: https://doi.org/10.21203/rs.3.rs-23490/v1

License: (c) (1) This work is licensed under a Creative Commons Attribution 4.0 International License. Read Full License

Version of Record: A version of this preprint was published at Journal of Medical Education and Curricular Development on January 1st, 2020. See the published version at https://doi.org/10.1177/2382120520957648. 


\section{Abstract}

\section{Background}

Inter-professional collaboration (IPC) is an important prerequisite for successful patient care. Even though inter-professional education (IPE) is increasingly common in undergraduate medical education, few IPE approaches explicitly address the IPC among medical students and students of psychology. IPE videos can be used to give learners the opportunity to gather relevant knowledge from different professional perspectives. So far it has been unclear whether it is enough when the topic of the video itself is inter-professional or if it is necessary for experts from different professions explicitly to appear in the video.

\section{Methods}

In an online experiment, medical students watched one of two videos about Parkinson's disease (PD) and the care of PD patients. The information was either provided by protagonists from only one profession (i.e., physicians; mono-professional condition) or provided by protagonists from two different professions (i.e., physicians and a psychologist; inter-professional condition). Attitude toward inter-professional interaction and learning, evaluation of the entertaining and illustrative character of the video, attitude toward physicians and psychologists, importance of IPC, evaluation of psychological treatment support, and knowledge acquisition served as dependent variables.

\section{Results}

The analysis was based on 140 participants (74 in the mono-, 66 in the inter-professional condition). We found that the inter-professional video was perceived to be more entertaining than the mono-professional video $\left(\mathrm{t}_{(138)}=-2.227 ; p=0.028 ; d=0.38\right)$. The inter-professional video was also considered to be more illustrative $\left(\mathrm{t}_{(138)}=-6.269 ; p<0.001 ; d=1.06\right)$. Moreover, participants improved their attitude toward physicians by watching the video $\left(F_{(1,138)}=4.860, p<0.001, \eta_{\mathrm{p}}^{2}=0.11\right)$, but they did not change their attitude toward psychologists $(p=0.146)$. Participants who watched the inter-professional video considered IPC to be more important than participants who watched the mono-professional video $\left(\mathrm{t}_{(138)}=\right.$ -7.954; $p<0.001 ; d=1.354)$. Finally, the inter-professional video led to better performance in the knowledge test $\left(\mathrm{t}_{(138)}=-2.285 ; p=0.024 ; d=0.04\right)$.

\section{Conclusions}

Inter-professional videos showing explicitly the appearance of experts from different professions come along with several advantages. We discuss the implications of their application in educational practice.

\section{Trial registration}

The study was pre-registered on the pre-registration platform AsPredicted (aspredicted.org) before we began data collection (registration number: \#33143). 


\section{Background}

In the health-care sector, inter-professional collaboration (IPC) is an important requirement for patient safety (1) and for a high quality of patient care $(2,3)$. Therefore, the implementation of inter-professional training in medical education is continually more in demand (4-6). Many patients are in contact with representatives from several health professions, and especially many chronic patients have a need for both medical and psychological support. Patients with chronic diseases have often co-morbid illnesses as is the case for patients with Parkinson's disease (PD). PD "is associated with substantial physical and mental co-morbidity" (7, p. 1). The cardinal symptoms in PD are motor symptoms (tremor, bradykinesia, rigidity, and postural instability (8). In addition, PD is often associated with non-motor symptoms like autonomic dysfunctions (9) and/or psychiatric conditions like dementia, depression, and anxiety $(7,10)$. The treatment options are very complex and some, like deep brain stimulation (DBS), should be accompanied by an inter-professional team. DBS is a neurosurgical procedure used to alleviate symptoms of PD that involves the implantation of remote-controlled electrodes into certain brain areas (11). Since this surgery often comes along with alterations of affect (12), interprofessional treatment, including psychologists for example, is recommended (13). This example illustrates the importance of smooth IPC between medical doctors and psychologists in clinical practice.

Ward, Zagoloff, Rieck, and Robiner (14) have requested that "Psychologists must embrace their identity as health professionals and engage their learners in IPE [interprofessional education] so that the emerging cognitive schemata of healthcare that is developed includes the profession of psychology" ( $p$. 250). They are concerned that otherwise "healthcare teams and health professionals will not understand the value, roles, or potential contributions of psychologists in enhancing patient care outcomes." $(14, \mathrm{p}$. 250). At first glance, this concern seems rather surprising, as the medical and psychological professions are already somewhat associated. Psychologist have been working in medical schools in clinical (e.g., clinical intervention, diagnosis) as well as non-clinical fields (e.g. research, teaching) for more than 100 years (15). However, psychologists are not necessarily directly involved in IPE activities. The Interprofessional Education Collaborative, for example, developed their first report about the core competencies for collaborative practice in 2011 without involving psychologists at all. Only the updated version from 2016 integrated input from the American Psychological Association (16). Even though IPE settings are more and more common in undergraduate medical education, only a few IPE programs (e.g. 17-19) explicitly address the IPC among medical students and students of psychology. But there is great demand for IPE settings that integrate psychological and medical perspectives (20).

\section{Videos in medical education}

One approach toward integrating psychological and medical perspectives in an IPE context is the provision of educational videos. Videos are already widely used in medical education, because they are an illustrative teaching format (21) and are effective for teaching procedural knowledge (22). Learning videos in healthcare education are especially relevant for inter-professional learning, since recent findings indicate that a video format could be suitable for the acquisition of inter-professional competences (23). 
This can be explained on the basis of Bandura's social learning theory, especially with regard to the fact that observational learning is suitable for the acquisition of social behavior $(24,25)$ : The observation of successful communication among professionals from different fields could therefore have an impact on both the acquisition of knowledge and the attitude toward interprofessional work. Furthermore, videos provide learners with the opportunity to capture the big picture (26) and therefore to develop metacognitive competences that are relevant for IPC (27). At the same time, the protagonists' professional identity influences how information is processed by the viewers (28). Videos are relatively easy to implement and could provide the opportunity for students to experience medical and psychological role models in related clinical settings. In a previous study (23), medical and physiotherapy students watched the same video that was interprofessional in two ways: The topic truly required an interprofessional approach, and protagonists from two different health professions appeared as role models. So far it has been unclear whether it is sufficient for making viewers learn about IPC and improving their attitude toward IPC and the collaborating professions simply to have the topic of the video be interprofessional, or if it is additionally necessary to have experts from multiple different professions explicitly appear in the video. To answer this research question, we investigated the impact of two different presentation formats in a medical video that dealt with a highly interprofessional topic: The procedures and treatments for DBS surgery (29) for patients with PD. The two conditions differed only in the video's presentation format: While one video presented a surgical procedure and discussion about this surgery among physicians only (mono-professional condition), the other video used the same material but additionally included a psychologist in the discussion (inter-professional condition).

\section{Hypotheses}

Based on social learning theory $(24,25)$ we hypothesized that presentation format (mono-professional vs. inter-professional) would have an impact on people's attitudes toward IPC. We hypothesized that the attitude of participants in the inter-professional condition toward inter-professional interaction (Hypothesis 1a) and inter-professional learning (Hypothesis 1b) would improve more than that of participants in the mono-professional condition.

Furthermore, we hypothesized that the presentation format would have an impact on the evaluation of the videos. An inter-professional video provides different perspectives, and explicitly observing rolemodels from different professions is more vivid and concrete. Thus, we hypothesized that the video in the inter-professional condition would be evaluated as more entertaining (Hypothesis 2a) and more illustrative of inter-professional collaboration (Hypothesis 2b) than in the mono-professional condition.

DBS is pertinent for psychologist as well as for physicians. As in both videos psychological as well as medical perspectives were presented, we hypothesized that the attitude of participants in both conditions would improve toward psychologists and toward physicians by watching the video (Hypothesis 3a). As the viewers in the inter-professional condition were explicitly confronted with both perspectives by observing role-models from both professions, we also hypothesized that this improvement would be stronger in the inter-professional condition than in the mono-professional condition (Hypothesis 3b). 
Moreover, we assumed that the presentation format would have an impact on the evaluation of the importance of IPC. Since the participants explicitly observed experts from different professions, we hypothesized that participants in the inter-professional condition would assess IPC's importance to be greater than participants in the mono-professional condition (Hypothesis 4).

Finally, we hypothesized that the presentation format would have an impact on the evaluation of psychological support during treatment. According to Xiao and Bavel (30), even implicit devaluation of a social outgroup disappeared when two groups were presented as being cooperative (instead of competitive). The inter-professional video format made the relevance of cooperation more evident than the mono-professional video. We hypothesized that participants in the inter-professional condition would evaluate psychological support during treatment more positively than participants in the monoprofessional condition (Hypothesis 5) after watching the video.

The perception of a video as entertaining might foster knowledge acquisition (31): A positive affective state helps encoding and storing information $(32,33)$ and thus may in turn facilitate knowledge acquisition. Based on these assumptions, we posed an open research question to examine whether there is an impact of presentation format on knowledge acquisition.

\section{Methods}

\section{Study design}

The study had a mixed design with presentation format (mono- vs. inter-professional) as between-group factor) and pre/post-comparison as within-group factor. Attitude toward interprofessional interaction and learning, evaluation of the entertaining and illustrative character of the video, attitude toward physicians and psychologists, importance of IPC, evaluation of psychological treatment support, and knowledge acquisition served as dependent variables.

\section{Participants}

288 participants followed a link that was distributed by the mailing list of the online video platform Sectio chirurgica (www.sectio-chirurgica.de, 34). 202 of them completed the questionnaire, but 60 failed the manipulation check and two were not medical students. Data of 140 participants thus remained for analysis: 74 participants were in the mono-professional condition (40 females, 34 males) and 66 (41 females, 25 males) in the inter-professional condition (see Figure 1). The participants' medical training ranged between the first and the tenth semesters, with most of the participants in earlier semesters ( $M=$ $4.15 ; \mathrm{SD}=2.64)$. Their mean age was 23.13 years old $(S D=3.53)$.

\section{Procedure}

The study was conducted online in January, 2020. Prior to the video presentation participants completed a demographic questionnaire that asked for their education history, age, and gender. Participants' professional identification (35), attitude toward psychological and medical treatment, and rating of 
interprofessional learning and interaction $(36,37,38)$ were also measured. Participants then watched a shortened episode from the medical online video platform Sectio chirurgica about a DBS surgical procedure, but the video differed in the two conditions. In the mono-professional condition the video showed the essential elements of the procedure and two physicians talking about DBS for PD. In the interprofessional condition, a psychologist's comments were additionally included. After watching the video, participants again filled in questionnaires on their attitude toward psychological and medical treatment and their rating of interprofessional learning and interaction. They were also asked to evaluate the video's entertainment value and its illustration of IPC. Then participants took a knowledge test. Finally, participants were informed about the aim of the study and the manipulation that was applied.

\section{Material and Measurements}

The videos were shortened versions of a 120-minute full episode, shortened to 8:22 minutes for the mono-professional condition and to 13:08 minutes for the inter-professional version. The interprofessional condition additionally presented a 4:44- minute interview with a psychologist conducted by a medical doctor.

\section{Attitude toward IPC}

Attitudes toward IPC interaction and learning were measured by the IP-interaction and IP-learning subscales (36-38) from the University of the West of England Interprofessional Questionnaire (UWE-IP). Both subscales comprised nine items each that were measured on 5-point Likert scales. Reliability of these scales were Cronbach's alpha $=0.712$ (IP-interaction) and Cronbach's alpha $=0.823$ (IP-learning) respectively.

\section{Evaluation of the video}

The participants' evaluation of the video was measured by two scales. The first scale rated the entertainment value of the video with six items. Reliability of this scale was Cronbach's alpha $=0.824$. The second scale rated how well the video illustrated IPC with three items with a reliability of Cronbach's alpha $=0.889$. All items were measured on 5-point Likert scales (see Table 1).

\section{Attitude toward the professions}

Participants' attitudes toward the medical and psychological professions was measured with an adapted version of Marteau, Dormandy, and Michie's scale (39) with four items on 7-point Likert scales, with a separate assessment of the medical and the psychological professions (see Table 2). Reliability of this scale was Cronbach's alpha $=0.948$.

\section{Evaluation of the importance of IPC}

A scale was created to evaluate the importance of IPC for DBS. This scale contained six items on a 5point Likert scale (see Table 3). Reliability of this scale was Cronbach's alpha $=0.815$. 


\section{Evaluation of psychological support during treatment}

A scale with four items was created to evaluate the psychological support during treatment. Reliability of these scales were Cronbach's alpha $=0.675$. Due to this low level of reliability, we did not include this scale in the analysis. Hypothesis 5 could therefore not be tested.

\section{Knowledge acquisition}

In the knowledge test the participants had to judge seven statements as correct or incorrect. In addition, they were asked two multiple choice questions (see Table 4). For each question the level of confidence for each answer was also rated on a scale ranging from 1 (very unconfident) to 6 (very confident). To reduce the probability of guessing, a balanced negative marking method was used (40) where each answer was analyzed for correctness $(+1=$ correct answer; $-1=$ wrong answer $)$ and multiplied with the confidence score.

\section{Statistical analysis}

We performed data analysis using IBM SPSS 22 for Windows (IBM Corp., Armonk, NY). Internal consistency of all scales was determined by calculating Cronbach's alpha (a). We tested age distribution with a t-test and gender distribution with a chi-squared test. To test the assumptions of Hypotheses 1 and 3, mixed-design ANOVAs with condition as between-group factor and the pre/post-comparison as withingroup factor were calculated. To test Hypotheses 2 and 4, as well as for the open research question, independent samples t-tests were used.

All data are reported as means (M) \pm standard deviations (SD), unless otherwise noted. The level of significance was set at $p<0.050$. Cohen's $d$ and partial eta squared $\left(\eta^{2}\right)$ are reported as indicators of effect size.

\section{Ethics statement}

This research was performed in accordance with the Declaration of Helsinki. The study had full approval by the ethics committee of the Leibniz-Institut für Wissensmedien (approval number: LEK 2017/035). All participants took part voluntarily and anonymously. They gave written informed consent and were informed about privacy protection, their right to terminate participation at any time without disadvantages, and about the general purpose of the study.

\section{Results}

\section{Equivalence of student groups}

We controlled the age and gender distributions of the two conditions. They did not differ from each other in age $\left(t_{(138)}=0.406 ; p=0.686\right)$ or gender $\left(\chi^{2}=3.457 ; p=0.063\right)$. 


\section{Attitude toward IPC}

Hypothesis $1 \mathrm{a}$ stated that the attitude of participants in the inter-professional condition toward interprofessional interaction would improve more than that of participants in the mono-professional condition. The data did not support this hypothesis. We found only a significant main effect for time (Pre: $\mathrm{M}=3.48, \mathrm{SD}=0.56$; Post: $\left.\mathrm{M}=3.59, \mathrm{SD}=0.51 ; F_{(1,138)}=5.176, p<0.024 ; \eta_{\mathrm{p}}^{2}=0.036\right)$, but no significant interaction effect between time and condition $(p=0.223)$.

Hypothesis $1 \mathrm{~b}$ assumed that the students' attitudes toward IPC learning would improve by observing the inter-professional video. Again, there was a significant effect for time (Pre: $M=3.65, S D=0.74 ;$ Post: $M=$ $\left.3.78, \mathrm{SD}=0.66 ; F_{(1,138)}=6.415, p=0.012, \eta_{\mathrm{p}}^{2}=0.044\right)$, but no significant interaction effect for time $\mathrm{x}$ condition $\left(F_{(1,138)}=0.219, p=0.641\right)$.

\section{Evaluation of the video}

We hypothesized that the video in the inter-professional condition would be evaluated as more entertaining (Hypothesis 2a) and more illustrative of IPC (Hypothesis $2 b$ ) than in the mono-professional condition. Hypothesis 2a was supported by the data; participants of the inter-professional condition evaluated the video as more entertaining $(M=2.65$; $S D=0.45)$ than participants of the monoprofessional condition $\left(\mathrm{M}=2.47 ; \mathrm{SD}=0.49 ; \mathrm{t}_{(138)}=-2.227 ; p=0.028 ; d=0.38\right)$.

Hypothesis $2 \mathrm{~b}$ was supported by the data as well. The inter-professional video was reported to be more illustrative of IPC $(M=3.20 ; S D=1.03)$ than the mono-professional video $\left(M=2.17 ; S D=0.92 ; t_{(138)}=\right.$ $-6.269 ; p<0.001 ; d=1.06)$.

\section{Attitude toward the professions}

We hypothesized that the attitudes of participants in both conditions would improve toward psychologists and toward physicians by watching the video (Hypothesis 3a). We also assumed that this improvement would be stronger in the inter-professional condition than in the mono-professional condition (Hypothesis 3b).

The data partly supported Hypothesis 3a. The attitudes of participants did improve toward physicians by watching the video (Pre: $M=6.48, S D=0.75$; Post: $M=6.75, S D=0.55 ; F_{(1,138)}=4.860, p<0.001, \eta_{p}^{2}=$ 0.11 ), but their attitudes toward psychologists did not change (Pre: $M=2.22, S D=0.55$; Post: $M=2.41$, $\left.S D=1.66 ; F_{(1,138)}=2.135, p=0.146\right)$. The assumptions of Hypothesis $3 b$ were not supported by the data: Participants' positive attitudes toward physicians increased more strongly in the mono-professional condition $\left(F_{(1,138)}=4.151, p=0.044, \eta_{p}^{2}=0.03\right)$ than in the inter-professional condition (see Figure 2 ). No interaction-effect was found for attitude toward psychologists $\left(F_{(1,138)}=0.392, p=0.532\right)$.

\section{Evaluation of the importance of IPC}


We hypothesized that participants in the inter-professional condition would assess the importance of IPC for DBS to be greater than participants in the mono-professional condition (Hypothesis 4). The data supported this hypothesis. Participants who watched the inter-professional video considered IPC to be more important $(M=4.18 ; S D=0.48)$ than participants who watched the mono-professional video $\left(\mathrm{M}=3.53 ; \mathrm{SD}=0.48 ; \mathrm{t}_{(138)}=-7.954 ; p<0.001 ; d=1.35\right)$.

\section{Knowledge acquisition}

After watching the video, participants in the inter-professional condition performed better in the knowledge test $(M=8.32 ; S D=10.45)$ than those in the mono-professional condition $(M=4.25 ; S D=$ $\left.10.54 ; \mathrm{t}_{(138)}=-2.285 ; p=0.024 ; d=0.04\right)$.

\section{Discussion}

Medical IPE videos can be used independently of time and location to give learners the opportunity to gather relevant knowledge from different professional perspectives (41). We investigated whether it is sufficient if the topic of the video itself is inter-professional (though being presented by representatives of only one profession), or if it is necessary for experts from different professions explicitly to appear in the video. The data collected in this study supported some but not all of our hypotheses. We therefore cannot provide final answers to the research questions, though some of our findings are valuable. On the one hand, our findings support the idea that the explicit appearance of experts from different professions is superior to a mono-professional presentation. We found that the inter-professional video was perceived to be more entertaining and more illustrative. This finding is in line with other studies that found that students perceived learning under the supervision of experts from professions other than their own to be rewarding and beneficial (42). Such experiences also open new perspectives for learners at all levels (43). A previous study (31) found that the acquisition of clinical knowledge was mediated by the entertainment aspect of a video. In the study presented here we also found that participants in the inter-professional condition learned more than the participants in the mono-professional condition. In addition, we found that participants in the inter-professional condition perceived IPC in the clinical context of DBS to be more important than participants in the mono-professional condition. This is an encouraging finding, as the main aim of IPE interventions is to foster IPC in clinical practice. However, based on this result, we cannot go so far as to say that the students' attitudes would actually result in more inter-professional behavior in clinical practice.

On the other hand, we have findings that did not support the idea that inter-professional videos are better for teaching inter-professional perspectives. Regarding the participants' attitudes toward IPC learning and IPC interaction, we found no superiority of the inter-professional video compared to the monoprofessional video. Both videos were equally effective in fostering positive attitudes toward IPC learning and IPC interaction. Based on this finding, it seems that for fostering positive attitudes toward IPE, the most important aspect is that the topic of the video itself is inter-professional. 
In the inter-professional condition, participants' attitudes toward physicians improved less than in the mono-professional condition. In addition, we found that the video presentation only affected participants' attitude toward physicians, but not toward psychologists. This result is in contrast to prior findings that showed that medical students tend to depreciate other professionals when being confronted with interprofessional collaboration situations $(37,44)$.

Moreover, we did not find any impact of the experimental condition on the evaluation of psychological support during treatment. Since all the participants were medical students, these findings must be interpreted with caution. We do not know to what extent their own professional identity had an impact on the findings. Research has shown that members of one's own social group are often rated more favorably than members of other groups (45). In future studies, medical as well as psychological students should participate to control for the influence of participants' own professional identity.

\section{Limitations}

Although we consider our study to be an important contribution to the development and use of IPE videos in medical education, we need to discuss some limitations. First, all of the participants were medical students, and most of them were at an early stage of their training. Thus, we cannot generalize these results to other healthcare-education populations. Moreover, although the videos showed experts from different professions contributing their profession-specific knowledge and perspectives, they did not really interact with each other. Future studies should make use of other topics that display a more obvious interaction among different healthcare professions.

Finally, it is still unclear how well these effects on inter-professional learning would transfer to interprofessional everyday practice. Findings which are reported about this question are based on active IPE programs and show rather low or inconsistent results $(2,3,46-48)$. For future research, long-term studies are required to investigate to what extent IPE videos can affect real clinical outcomes.

\section{Conclusions}

This experimental study compared two possible IPE video formats to foster inter-professional learning in healthcare education. To represent IPC, we used the example of collaboration among physicians and psychologists. An advantage of IPE videos is that they are easy to implement in existing curricula, or they can be used as add-ons to curricular training, to illustrate and foster inter-professional collaboration. Although the data did not support all of the hypotheses, we may conclude that the explicit appearance of experts from different professions in an educational video had a positive influence on how medical students perceived the video and how they evaluated IPC. The inter-professional video also led to better performance on a knowledge test of the medical students in this study.

\section{Abbreviations}

DBS: Deep brain stimulation 
IPC: Interprofessional collaboration

IPE: Interprofessional education

PD: Parkinson's disease

\section{Declarations}

Ethics approval and consent to participate

This research was performed in accordance with the Declaration of Helsinki. This study had full approval by the ethics committee of the Leibniz-Institut für Wissensmedien (approval number: LEK 2017/035). All participants took part voluntarily and anonymously. They gave written informed consent and were informed about privacy protection, their right to terminate participation at any time without disadvantages, and about the general purpose of the study.

Consent for publication

Not applicable

Availability of data and material

Data are available on request to the authors.

The dataset and analysis for the current study are available at OPEN ICPSR, https://www.openicpsr.org/openicpsr/project/118961/version/V1/view/

Competing interests

The authors declare that they have no competing interests.

Funding

The research reported here was supported by a grant from German Federal Ministry of Education and Research; Grant number: 16DHL1030. The authors also acknowledge support by Deutsche Forschungsgemeinschaft and Open Access Publishing Fund of Tuebingen University. None of these funding bodies has exerted any influence on the design of the study and collection, analysis, interpretation of data and on writing the manuscript.

Authors' contributions

JG, MB and JK contributed to the conception and design of this study. TS and BH made substantial contributions to the acquisition of data. JG performed the statistical analysis. JG, MB and JK were responsible for drafting the article. TS and $\mathrm{BH}$ contributed to its critical revision. All authors approved the final manuscript for publication. 
Acknowledgements

We would like to thank the Department of General Practice and Health Services Research (University Hospital Heidelberg, Heidelberg, Germany) for the permission for the use of the German version of the UWE-IP questionnaire and Simone Korger for her assistance in data collection.

\section{References}

1. Gill A, Cowart J, Hatfield C, Dello Stritto R, Landrum P, Ismail N, et al. Patient Safety Interprofessional Training for Medical, Nursing, and Pharmacy Students. MedEdPORTAL Publ. 2017;

2. Guraya SY, Barr H. The effectiveness of interprofessional education in healthcare: a systematic review and meta-analysis. Kaohsiung J Med Sci. 2018;34(3):160-5.

3. Solomon P, Baptiste S, Hall P, Luke R, Orchard C, Rukholm E, et al. Students' perceptions of interprofessional learning through facilitated online learning modules. Med Teach. 2010;

4. Barr H, Freeth D, Hammick M, Koppel I, Reeves S. The evidence base and recommendations for interprofessional education in health and social care. J Interprof Care. 2006;

5. Walkenhorst U, Mahler C, Aistleithner R, Hahn EG, Kaap-Fröhlich S, Karstens S, et al. Positionspapier GMA-Ausschuss-Interprofessionelle Ausbildung in den Gesundheitsberufen. Gms Z Med Ausbild. 2015;32(2):95424-42.

6. Wilhelmsson M, Pelling S, Ludvigsson J, Hammar M, Dahlgren LO, Faresjö T. Twenty years experiences of interprofessional education in Linköping - Ground-breaking and sustainable. J Interprof Care. 2009;

7. McLean G, Hindle J V., Guthrie B, Mercer SW. Co-morbidity and polypharmacy in Parkinson's disease: Insights from a large Scottish primary care database. BMC Neurol. 2017;

8. Jankovic J. Parkinson's disease: Clinical features and diagnosis. Journal of Neurology, Neurosurgery and Psychiatry. 2008.

9. Micieli G, Tosi P, Marcheselli S, Cavallini A. Autonomic dysfunction in Parkinson's disease. Neurol Sci. 2003;

10. Landau S, Harris V, Burn DJ, Hindle J V., Hurt CS, Samuel M, et al. Anxiety and anxious-depression in Parkinson's disease over a 4-year period: A latent transition analysis. Psychol Med. 2016;

11. Bronstein JM, Tagliati M, Alterman RL, Lozano AM, Volkmann J, Stefani A, et al. Deep brain stimulation for Parkinson disease an expert consensus and review of key issues. Archives of Neurology. 2011.

12. Synofzik M, Schlaepfer TE. Stimulating personality: Ethical criteria for deep brain stimulation in psychiatric patients and for enhancement purposes. Biotechnology Journal. 2008.

13. Morishita T, Fayad SM, Higuchi M aki, Nestor KA, Foote KD. Deep Brain Stimulation for Treatmentresistant Depression: Systematic Review of Clinical Outcomes. Neurotherapeutics. 2014. 
14. Ward W, Zagoloff A, Rieck C, Robiner W. Interprofessional Education: Opportunities and Challenges for Psychology. J Clin Psychol Med Settings. 2018;

15. Robiner WN, Dixon KE, Miner JL, Hong BA. Psychologists in medical schools and academic medical centers: Over 100 years of growth, influence, and partnership. Am Psychol. 2014;

16. Interprofessional Educational Collaborative, Practice IC, Values U. Core Competencies for Interprofessional Collaborative Practice: 2016 Update. Interprofessional Educ Collab. 2016;

17. Köllner V, Foltin Y, Speidel V, Müller AK, Jäger J. Anamnesegruppen als Einstieg in die Gesprächsführung und Arzt - Patient-Beziehung. Medizinische Welt. 2016.

18. Anderson GL, Lovejoy DW. Predoctoral training in collaborative primary care: An exam room built for two. Prof Psychol Res Pract. 2000;

19. Bridges DR, Davidson RA, Odegard PS, Maki I V., Tomkowiak J. Interprofessional collaboration: Three best practice models of interprofessional education. Med Educ Online. 2011;

20. Cubic B, Mance J, Turgesen JN, Lamanna J. Interprofessional education: Preparing psychologists for success in integrated primary care. J Clin Psychol Med Settings. 2012;

21. Welsher A, Grierson LEM. Enhancing technical skill learning through interleaved mixed-model observational practice. Adv Heal Sci Educ. 2017;

22. Domuracki K, Wong A, Olivieri L, Grierson LEM. The impacts of observing flawed and flawless demonstrations on clinical skill learning. Med Educ. 2015;

23. Grosser J, Bientzle M, Shiozawa T, Hirt B, Kimmerle J. Observing interprofessional collaboration: Impact on attitude and knowledge acquisition.

24. Bandura A. Social Cognitive Theory of Mass Communication. Media Psychology. 2001.

25. Bandura A. Social foundations of thought and action: a social cognitive theory / Albert Bandura. Englewood Cliffs, N.J: Prentice-Hall. 1986.

26. Hober C, Bonnel W. Student Perceptions of the Observer Role in High-Fidelity Simulation. Clin Simul Nurs. 2014;

27. Clark PG. What would a theory of interprofessional education look like? Some suggestions for developing a theoretical framework for teamwork training. J Interprof Care. 2006;

28. König L, Jucks R. Hot topics in science communication: Aggressive language decreases trustworthiness and credibility in scientific debates. Public Underst Sci. 2019;

29. Machado A, Rezai AR, Kopell BH, Gross RE, Sharan AD, Benabid AL. Deep brain stimulation for Parkinson's disease: Surgical technique and perioperative management. Mov Disord. 2006;

30. Xiao YJ, Van Bavel JJ. Sudden shifts in social identity swiftly shape implicit evaluation. J Exp Soc Psychol. 2019;83:55-69. doi: 10.1016/j.jesp.2019.03.005

31. Grosser J, Bientzle M, Shiozawa T, Hirt B, Kimmerle J. Acquiring clinical knowledge from an online video platform: A randomized controlled experiment on the relevance of integrating anatomical information and clinical practice. Anat Sci Educ. 2018;12(5):478-84. doi: 10.1002/ase.1841 
32. Fredrickson BL. What good are positive emotions? Rev Gen Psychol. 1998;2(3):300-19.

33. Isen AM. A role for neuropsychology in understanding the facilitating influence of positive affect on social behavior and cognitive processes. In: Lopez SJ, Snyder CR, editors. Oxford handbook of positive psychology. New York: Oxford University Press; 2009. p. 503-18.

34. Hirt B, Shiozawa T, Herlan S, Wagner H-J, Küppers E. Surgical prosection in a traditional anatomical curriculum-Tübingens' Sectio chirurgica. Ann Anatomy-Anatomischer Anzeiger. 2010;192(6):34954.

35. Adams K, Hean S, Sturgis P, Clark JM. Investigating the factors influencing professional identity of first-year health and social care students. Learn Heal Soc Care. 2006;5(2):55-68. doi: 10.1111/j.14736861.2006.00119.x

36. Mahler C, Berger S, Pollard K, Krisam J, Karstens S, Szecsenyi J, et al. Translation and psychometric properties of the German version of the University of the West of England Interprofessional Questionnaire (UWE-IP). J Interprof Care. 2017;31(1):105-9. doi: 10.1080/13561820.2016.1227964

37. Pollard KC, Miers ME, Gilchrist M. Collaborative learning for collaborative working? Initial findings from a longitudinal study of health and social care students. Health Soc Care Community. 2004;12(4):346-58.

38. Pollard KC, Miers ME, Gilchrist M. Collaborative learning for collaborative working? Initial findings from a longitudinal study of health and social care students. Health Soc Care Community. 2004;12(4):346-58. doi: 10.1111/j.1365-2524.2004.00504.x

39. Marteau TM, Dormandy E, Michie S. A measure of informed choice. Heal Expect. 2001;4(2):99-108. doi: 10.1046/j.1369-6513.2001.00140.x

40. Gardner-Medwin AR, Gahan M. Formative and Summative Confidence-Based Assessment. Proc. 7th International Computer-Aided Assessment Conference. 2003.

41. van Es EA, Tunney J, Goldsmith LT, Seago N. A framework for the facilitation of teachers' analysis of video. J Teach Educ. 2014;65(4):340-56. doi: 10.1177/0022487114534266

42. Chipchase L, Allen S, Eley D, McAllister L, Strong J. Interprofessional supervision in an intercultural context: A qualitative study. J Interprof Care. 2012;26(6):465-471. doi:

10.3109/13561820.2012.718813

43. Ruiz MG, Ezer H, Purden M. Exploring the nature of facilitating interprofessional learning: Findings from an exploratory study. J Interprof Care. 2013;27(6):489-95. doi: 10.3109/13561820.2013.811640

44. Hertweck ML, Hawkins SR, Bednarek ML, Goreczny AJ, Schreiber JL, Sterrett SE. Attitudes toward interprofessional education: Comparing physician assistant and other health care professions students. J Physician Assist Educ. 2012;23(2):8-15.

45. Sollami A, Caricati L, Mancini T. Attitudes towards Interprofessional Education among Medical and Nursing Students: the Role of Professional Identification and Intergroup Contact. Curr Psychol. 2018;37(4):905-12. doi: 10.1007/s12144-017-9575-y

46. McCutcheon LRM, Alzghari SK, Lee YR, Long WG, Marquez R. Interprofessional education and distance education: A review and appraisal of the current literature. Currents in Pharmacy Teaching 
and Learning. 2017;9(4):729-36. doi: 10.1016/j.cptl.2017.03.011

47. Karim R, Ross C. Interprofessional education (IPE) and chiropractic. J Can Chiropr Assoc. 2008;52(2):76-78.

48. Reeves S, Pelone F, Harrison R, Goldman J, Zwarenstein M. Interprofessional collaboration to improve professional practice and healthcare outcomes. Cochrane Database Syst Rev. 2017;6. doi: 10.1002/14651858.CD000072.pub3

\section{Tables}

Table 1 - Video evaluation scale

\section{Entertainment}

$\begin{array}{lllll}1 & 2 & 3 & 4 & 5 \\ \begin{array}{l}\text { (I don't } \\ \text { agree at } \\ \text { all) }\end{array} & & & & \begin{array}{l}\text { (I fully } \\ \text { agree) }\end{array}\end{array}$

I thought the video was entertaining.

I found the video exciting.

The video was thrilling.

I found the video diverting.

I had fun watching this video.

I was fascinated by this video.

\section{Illustrative character}

The video provided good insight into the cooperation of different professions.

Watching the video prepared me for an interprofessional cooperation with psychologists.

I think the video gave me a better understanding of the actions and thinking of other professionals.

\section{Table 2 - Attitude toward the professions}


I think that in the in the context of DBS, the support of the patient by a doctor / a psychologist is....

\begin{tabular}{|c|c|c|c|c|c|c|c|c|}
\hline & 1 & 2 & 3 & 4 & 5 & 6 & 7 & \\
\hline Useless & $\bullet$ & 0 & 0 & $\bullet$ & 0 & $\bullet$ & 0 & useful \\
\hline Harmful & $\bullet$ & 0 & 0 & $\bullet$ & 0 & $\bullet$ & 0 & beneficial \\
\hline unimportant & 0 & 0 & 0 & 0 & 0 & $\bullet$ & 0 & important \\
\hline a bad thing & 0 & 0 & 0 & 0 & 0 & $\bullet$ & 0 & a good thing \\
\hline
\end{tabular}

Table 3 - Evaluation of the importance of IPC

(I don't agree at all) 1

$\begin{array}{llll}2 & 3 & 4 & \begin{array}{l}5(\mathrm{I} \\ \text { fully } \\ \text { agree })\end{array}\end{array}$

The patient's preparation for a neurosurgical intervention should be carried out both by physicians and psychologists.

A close cooperation between psychologists and doctors is important for patient's complete information.

Inter-professional cooperation between psychologists and doctors is beneficial for an ideal therapeutic outcome.

The involvement of psychological specialists to care for patients during treatment makes sense for patients.

The involvement of psychological specialists to care for patients during treatment is helpful for patients.

Patients who have received DBS should be monitored afterwards by psychologists.

\section{Table 4 - Knowledge test}


multiple choice
Multiple answers can be correct
Confidence rating

very unconfident --_- very confident
As imaging techniques are not sufficient for optimal trajectories planning, additional methods need to be applied. Which ones?
The cardinal symptoms of Parkinson's disease are called the triad. These include:

\section{Electrophysiological $\quad \square$} methods

Audiometric

methods

Electroneurographic [

methods

Transcranial

Doppler methods

]

$\begin{array}{llllll}1 & 2 & 3 & 4 & 5 & 6\end{array}$

\begin{tabular}{lc} 
Amnesia & $\square$ \\
\hline Akinesia & $\square$ \\
\hline Aphasia & $\square$ \\
\hline Mutism & $\square$ \\
\hline Rigor & $\square$
\end{tabular}

Tremor

प

forced choice

About $80 \%$ of patients suffering from Parkinson's disease are treated with DBS.
Please choose

Correct

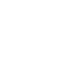

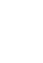

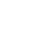

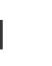

About $80 \%$ of deep brain stimulation surgeries are performed on patients with Parkinson's disease.

To avoid cerebral damage, gyri should be avoided in trajectory planning.

The symptoms of akinesia and rigor can easily be reduced by stimulating fibrous tracts in the zona incerta.

Depression in Parkinson's disease is caused by cerebral cell death. stimulating the anterior

प 口

wrong not at all -___-_-absolutely

Confidence rating 
subthalamic nucleus.

Figures

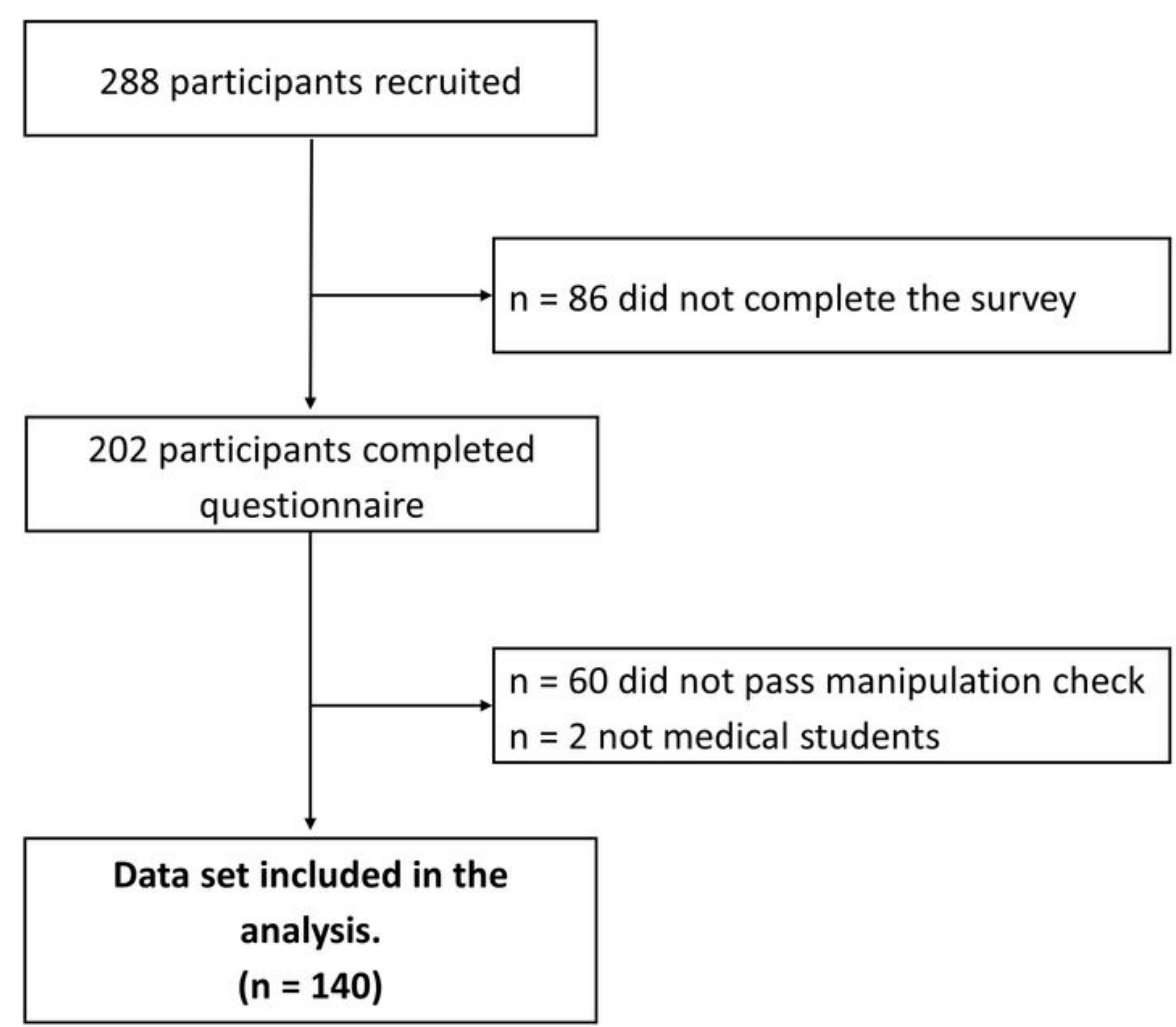

\section{Figure 1}

Flow diagram of study design 


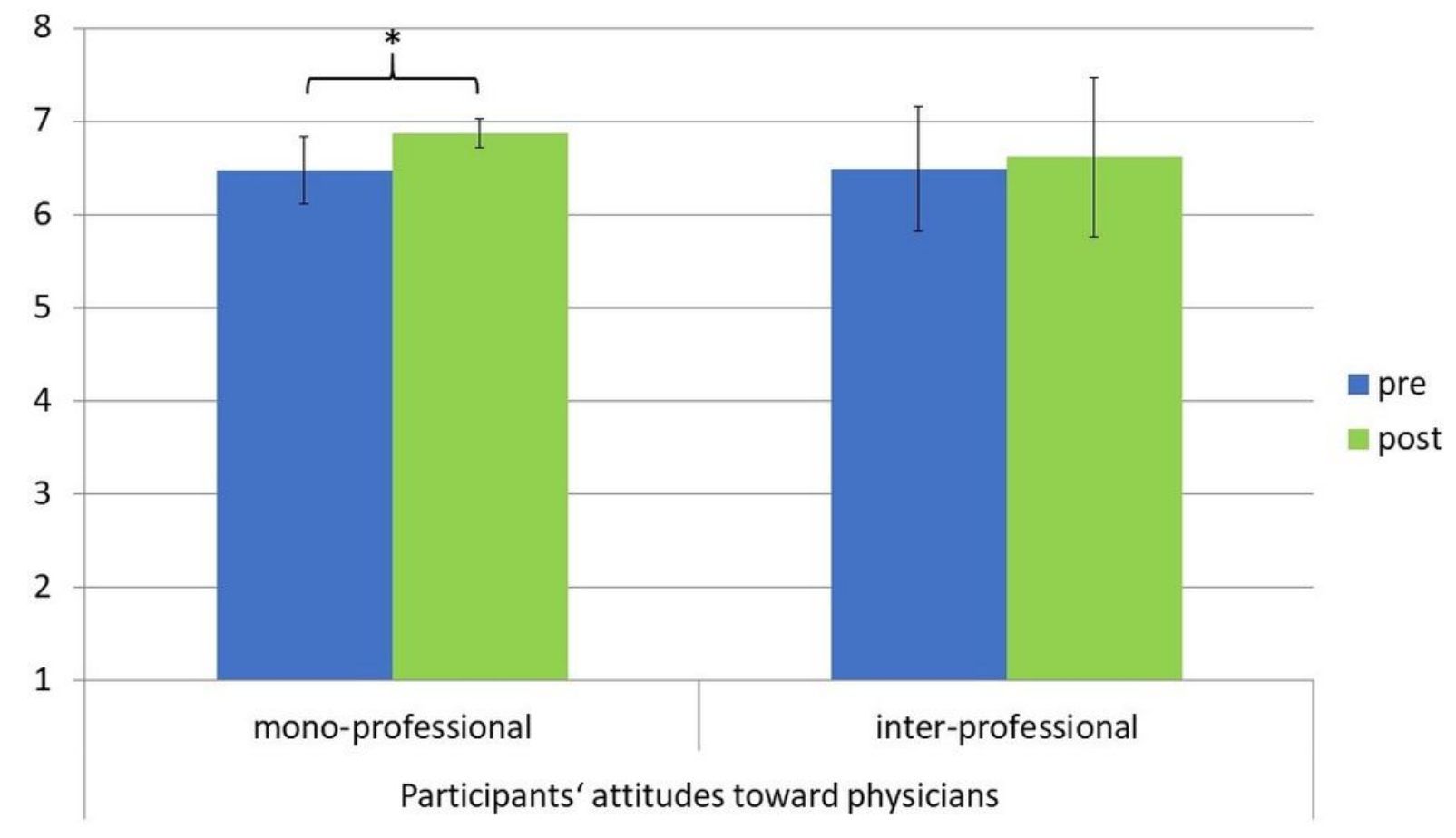

Figure 2

Participants' attitudes toward physicians before and after watching the video. Standard errors are represented by the error bars attached to each column. 